\title{
Delayed MRI Findings in Herpes simplex Encephalitis
}

\author{
Yun Jiang, Liya Tang, Ping Gao, Shaosen Qin, Jingwen Jiang
}

Can. J. Neurol. Sci. 2011; 38: 155-157

Herpes simplex encephalitis (HSE) is one of the most common forms of encephalitis, accounting for approximately $10 \%$ of all cases of encephalitis. Herpes simplex virus -1 (HSV) causes more than $90 \%$ of HSE cases in adults.

Herpes simplex encephalitis is a life-threatening disease. Following the onset of neurological symptoms, most patients rapidly progress to confusion, lethargy or coma within a few days. When initiated early, acyclovir treatment greatly benefits the prognosis, reducing the mortality rate from $70 \%$ to $25 \% 1,2$. Therefore, a rapid and reliable diagnosis is very important. Cerebral spinal fluid (CSF) analysis of viral DNA, using polymerase chain reaction (PCR), a technique highly sensitive and specific for the rapid diagnosis of HSE, appears positive for HSE between 3 and 12 days following the onset of neurological symptoms $^{3,4}$. The old standard is viral culture or brain biopsy, but that PCR, with its excellent sensitivity and feasibility, is considered diagnostic and definitive in most clinical settings. Brain magnetic resonance imaging (MRI) is also very sensitive in showing characteristic changes in temporal lobe, frontobasal lobe and occasionally in the insular cortex and cingular gyri in $\mathrm{HSE}^{5-8}$. In most reported cases, MRI revealed brain abnormalities within one week after the onset of $\operatorname{HSE}^{6-8}$. The characteristic and sensitive findings of MRI help the early diagnosis of HSE. Here we report an atypical HSE case, which showed subacute clinical process and delayed MRI characteristic abnormalities.

\section{Case Report}

A 56 year-old previous healthy man was admitted to the Emergency Department shortly after a focal seizure of his left cheek and left arm accompanied by a transient loss of consciousness. He was nauseated and vomiting and complained of fatigue and forehead pain. The patient had no fever and the neurological assessment was normal. Blood count revealed 11.24 $\times 10^{9} / \mathrm{L}$ white blood cells (WBC) with $58 \%$ neutrophils. The routine biochemical analyses were normal. The electroencephalogram showed poor adjustment of $\beta$ wave amplitude. Brain MRI (Siemens, 1.5T) was normal (Figure A, B). There was no recurrence of seizure and the next day he was discharged from the hospital. He developed progressive memory deficits. On the 13 th day, he felt pain in the upper back and shoulder concurrent with severe dizziness. He had a temperature of $37.6^{\circ} \mathrm{C}$ and was re-admitted into the hospital. He was very irritable with hallucinations and disorientation. On the day of re-admission, he experienced three bouts of generalized tonic-clonus seizure. He rapidly progressed to lethargy. Electro-encephalogram revealed single spike wave in the bilateral frontal and the left temporal regions with increased $\theta$ waves in the right occipital and temporal regions. The serum autoantibodies, including antinuclear antibodies, anti-double strand DNA, antineurotrophic cytoplastic antibodies, and anti-Ro/SSA and anti-La/SSB, were negative and thyroid function was in the normal range. On the 14 th day, a lumbar puncture showed normal cell count with mildly increased protein content $(687 \mathrm{mg} / \mathrm{L})$. Cerebral spinal fluid IgG and IgM antibodies of several viruses, including HSV1 , zoster herpes virus, and cytomegalovirus, were negative. The analysis of viral DNA PCR was not available in the first CSF test. Herpes simplex encephalitis was considered. He was treated with phenytoin and intravenous acyclovir $10 \mathrm{mg} / \mathrm{kg}$, every eight hours. On the 15 th day, the fluid-attenuated inversion recovery (FLAIR) and T1-weighted MRI after administration of GdDTPA were performed and again, no abnormality was identified (Figure C, D). The patient continued to suffer from seizures three to four times/day. He deteriorated rapidly and on the 17th day, after three continuous seizures, he became comatose. He developed severe aspiration pneumonia and was placed on a ventilator. Broad spectrum antibiotic treatment was initiated. Because of the clinical deterioration, $5 \mathrm{mg}$ intravenous dexamethasone was administered every six hours. On the 23th day, the third MRI clearly revealed abnormal signals in both medial temporal lobe, mainly the left hippocampus, which displayed hypointensity on Tl-weighted images and hyperintensity on T2-weighted, diffusion-weighted (Figure E) and FLAIR (Figure F) images. On the 27 th day, lumbar puncture revealed 31 white blood cells $/ \mathrm{ml}$ consisting of $79 \%$ polymorphonuclear cells and a protein level of $731 \mathrm{mg} / \mathrm{L}$. Herpes simplex virus -1 PCR was negative, and CSF HSV-I antibodies were also negative. The acyclovir treatment continued for three weeks. The patient gradually recovered and the seizures disappeared. He regained consciousness and orientation. A third lumbar puncture was performed on the 42nd day and CSF cell count returned to normal and HSV-1 IgG was determined to be positive, while zoster herpes virus IgG, and cytomegalovirus IgG were negative. Herpes simplex virus -1 PCR remained negative. The diagnosis of HSE-1 was confirmed. The patient continued to improve. The follow-up MRI was done on the 52 nd day, and the abnormal signals in bilateral temporal lobes were present, but

\footnotetext{
From the Department of Neurology, Beijing Hospital, Beijing, China.

Received June 16, 2010. Final Revisions Submitred August 4, 2010. Correspondence to: Yun Jiang, Depatinent of Neurology, Beijung Hospital, Beijing 100730 , China.
} 

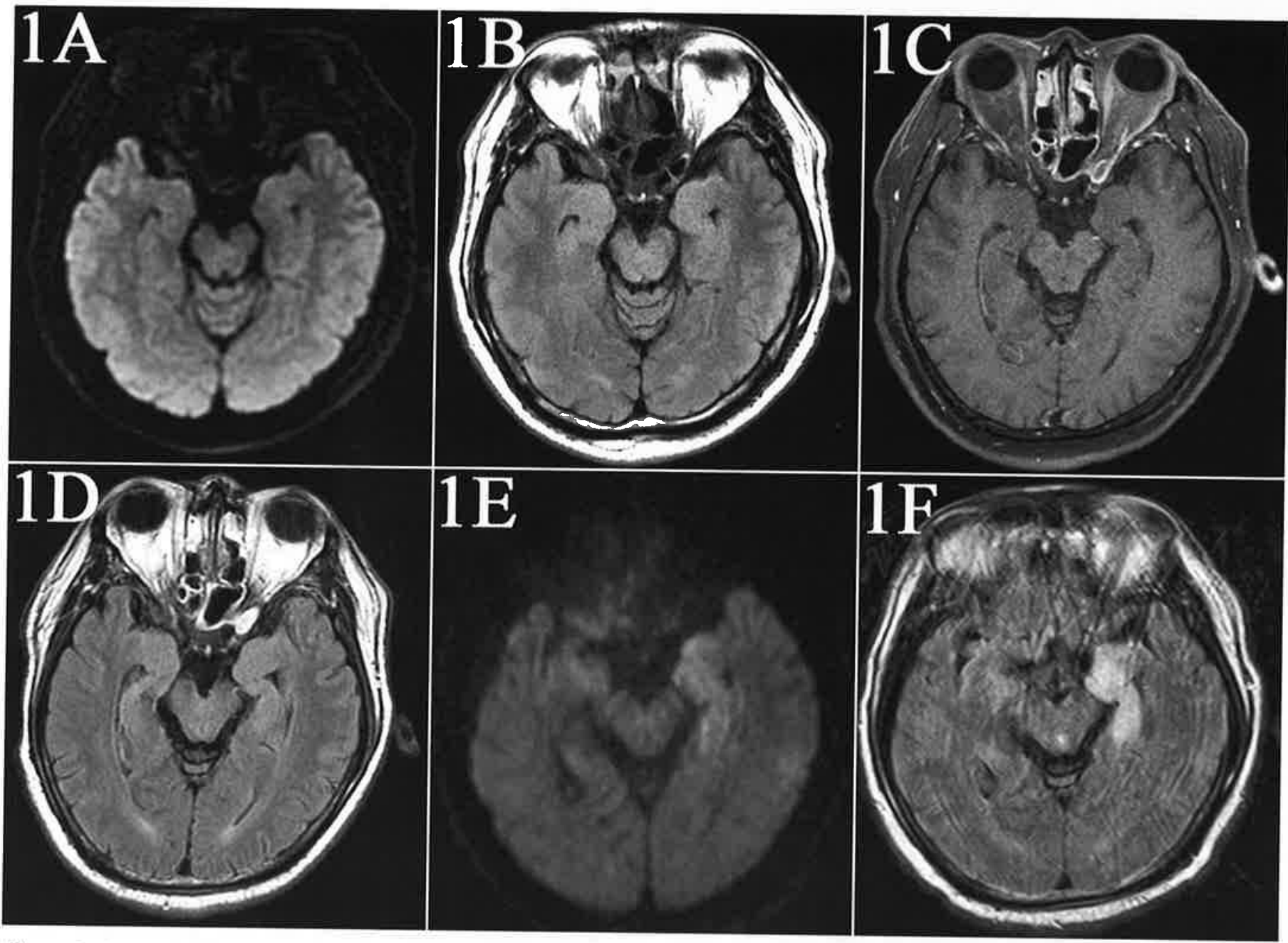

Figure: Serial cranial MRI scans of the HSE patient. On the second day following the first seizure, MRI was normal (A-Diffusion, B-FLAIR). On the I5th day, the contrasted-enhanced TI weighted images (C) and FLAIR (D) did not reveal apparent abnormality, either. On the 23th day, abnormal signals were present on both sides of medial temporal lobe, mainly in the left hippocampus with a small lesion in the right hippocampus, which displayed hyperintensity in Diffusion (E) and FLAIR $(F)$.

weakened (pictures not shown). The patient returned home with impaired memory, especially short-term memory.

\section{Discussion}

This patient was diagnosed with HSE by intrathecal synthesis of antibody against HSV- 1 . The case was characterized by several uncommon features. First, the initiation of HSE in this patient was subacute. He had a delay of 11 days after the first seizure, followed by apparent neurological and psychological manifestations, and then he deteriorated quickly. Second, he had a late increase of WBC in the CSF with polymorphonuclear cell predominance. Third, he had delayed positive MRI findings. This patient underwent serial MRI scans following the first seizure and apparent abnormality appeared on the 23rd day, but not on the 15 th. To our knowledge, negative MRI findings more than 15 days after the onset of neurological symptoms of HSE have not been reported previously. Hollinger et al reported normal MRI findings in a PCR confirmed HSE patient, where the patient was followed for 14 months ${ }^{9}$. However, during the acute phase, the last MRI was taken on Day $6 \%$. Our results indicate that abnormal MRI findings in HSE can occur as late as two to three weeks, and the cases with normal MRI findings in early stage must be followed more closely within the first month.

Magnetic resonance imaging is very sensitive in the detection of morphological abnormalities in acute HSE cases. Positive brain MRI is generally documented within one week of the HSE onset $^{5-8}$. Magnetic resonance imaging clearly shows the typical HSE lesions in the unilateral or bilateral temporal lobes, insular cortex, frontobasal lobes, which display hypointensity in $\mathrm{T} 1$, and hyperintensity in T2, Diffusion and FLAIR images ${ }^{5-8}$. It is of note that the Diffusion and FLAIR are the most sensitive radioimaging methods in detecting $\mathrm{HSE}^{7,8}$. In an immunodeficient patient, the abnormal MRI findings were found to occur earlier than PCR DNA tests in CSF. The MRI Diffusion revealed hyperintensity in the insular lobe on the 2nd day after neurological symptoms appeared, when PCR of HSV was still 
negative. Polymerase chain reaction of HSV was positive on the 5 th day $^{8}$. To exclude tumor and acute disseminated encephalomyelitis, we applied for the contrasted MRI, including FLAIR, 15 days after his first seizure. Fluid-attenuated inversion recovery is superior than $\mathrm{T} 1$ and $\mathrm{T} 2$ in detecting the early changes of HSE, and usually shows the lesions around one week after the onset of neurological symptoms ${ }^{6,8}$. The negative FLAIR findings 15 days after the first seizure indicated delayed abnormalities in brain MRI.

Cerebral spinal fluid PCR-test of HSV-1 is the gold standard in the diagnosis of HSE. It is reported that the PCR-positive period for HSE is from Day 3 to Day $12^{4}$. Polymerase chain reaction can be negative within two days of HSE onset ${ }^{4.8}$. The negative finding of CSF PCR in our patient could be due to the two weeks acyclovir treatment ${ }^{9}$. Positive PCR appeared in the acute stage and intrathecal IgG could be measured in the postacute stage. Intrathecal IgM/IgG were detectable 14 days after onset of the disease and persisted for several years. Generally, PCR is negative in the cases with detectable intrathecal $\mathrm{IgG}^{4}$. The sensitivity and specificity values of specific HSV $-1 \mathrm{lgG}$ assay are about $80 \%{ }^{10}$. The false positive mainly arises from the cross-reaction with varicella zoster antibodies ${ }^{11}$ In our patient, the CSF varicella zoster virus IgG was negative in the three tested time points. The specific HSV-I IgG in CSF in our patient turned from negative on Day 14 and 27 to positive on Day 42 , confirming the diagnosis of HSE. The etiological diagnosis of HSE is very important because other causes of limbic encephalitis, such as connective tissue disorders, paraneoplastic and voltage gated potassium channel associated limbic encephalitis, also selectively involve the hippocampus and other limbic structures ${ }^{12}$.

The reason for delayed MRI findings in our HSE patient is unclear. This patient had no seizure, previously. He had a subacute onset of HSE. After the first seizure, he had a plateau phase of 11 days, and then his condition worsened rapidly. The second negative MRI was taken on the 3rd day after the deterioration. On the 11 th day after the deterioration, MRI revealed characteristic medial temporal lobe lesions. The increase of CSF WBC occurred in parallel to the positive MRI findings. We propose that the subacute onset of HSE may account for the delayed MRI findings in this patient.

Herpes simplex encephalitis is a severe disease. The combination of MRI and CSF PCR-test promote the early diagnosis of HSE, However, the assessment of CSF-PCR and brain MRI at the onset of neurological symptoms can be negative. For those patients with clinically suspected HSE and negative initial findings in CSF and MRI, serial, repeated tests of CSF PCR, CSF IgM and IgG tests and MRI are required to confirm the diagnosis of HSE.

\section{ACKNOWLEDGEMENT}

The authors thank Dr. Joshua A. Gregory for his careful review of this manuscript and Saying Li, Department of Radiology, Beijing Hospital, for providing magnetic resonance images.

\section{REFERENCES}

1. Raschilas F, Wolff M, Delatour F, et al, Outcome of and prognostic factors for herpes simplex encephalitis in adult patients: results of a multicenter study. Clin Infect Dis. 2002 Aug 1:35(3):254-60.

2. Kamei S, Sekizawa T, Shiota H, et al. Evaluation of combination therapy using aciclovir and corticosteroid in adult patients with herpes simplex virus encephalitis. I Neurol Neurosurg
Psychiatry. 2005 Nov;76(11):1544-9.

3. Kennedy PG. Viral encephalitis. J Neurol. 2005 Mar;252(3):

4. Sauerbrei A, Eichhorn U, Hottenrott G, Wutzler P. Virological diagnosis of herpes simplex encephalitis. J Clin Virol. 2000 Jun;
17(1):31-6.

5. Gumus H, Kumandas S, Per H, Tahan F, Koklu E, Karakukcu M. Unusual presentation of herpes simplex virus encephalitis: bilateral thalamic involvement and normal imaging of early stage of the disease. Am J Emerg Med. 2007 Jan;25(1):87-9.

6. Gaviani P, Leone M, Mula M, et al. Progression of MRI abnormalities in herpes simplex encephalitis despite clinical improvement: natural history or disease progression? Neurol Sci. 2004 Jun;25(2): 104-7.

7. Kuker W, Nagele T, Schmidt F, Heckl S, Herrlinger U. Diffusionweighted MRI in herpes simplex encephalitis: a report of three cases. Neuroradiology. 2004 Feb;46(2):122-5.

8. Akyldz BN, Gumus H, Kumandas S, Coskun A, Karakukucu M, Yklmaz A. Diffusion-weighted magnetic resonance is better than polymerase chain reaction for early diagnosis of herpes simplex encephalitis: a case report. Pediatr Emerg Care. 2008 Jun;24(6): $377-9$.

9. Hollinger P, Matter L, Sturzenegger M. Normal MRI findings in herpes simplex virus encephalitis. J Neurol. 2000 Oct;247(10): 799-801,

10. Whitley RJ, Lakeman F. Herpes simplex virus infections of the central nervous system: therapeutic and diagnostic considerations. Clin Infect Dis. 1995 Feb;20(2):414-20.

11. Cinque P, Cleator GM, Weber T, Monteyne P, Sindic CJ, van Loon $\mathrm{AM}$. The role of laboratory investigation in the diagnosis and management of patients with suspected herpes simplex encephalitis: a consensus report. The EU Concerted Action on Virus Meningitis and Encephalitis. J Neurol Neurosurg Psychiatry. 1996 Oct;61(4):339-45.

12. Schott JM. Limbic encephalitis: a clinician's guide. Pract Neurol. 2006;86:143-53. 